\title{
Interface de doenças endocrinológicas com repercussões psiquiátricas: uma revisão de literatura
}

Interface of endocrinological diseases

with psychiatric repercussions: a literature review

Interfaz de enfermedades endocrinológicas con repercusiones psiquiátricas: una revisión de la literatura

Daniell de Siqueira Araújo Lafayette - https://orcid.org/0000-00029187-0532

Philip de Azevedo Costa Urquiza - https://orcid.org/0000-0002-97904502

Eveline Maria Cosmo de Brito - https://orcid.org/0000-0002-3228-0049

Silvia Poliana Guedes Alcoforado Costa - https://orcid.org/0000-0002$\underline{6478-3847}$

Amaury Cantilino - https://orcid.org/0000-0002-4382-1218

\section{RESUMO:}

Introdução: várias síndromes clínicas podem apresentam sintomas neurológicos e psiquiátricos. Objetivo: este artigo está focado em descrever as principais síndromes endócrinas que podem cursar com alterações psiquiátricas. Método: foi realizada uma revisão de literatura por meio de busca e análise de artigos em inglês no Pubmed com descritores e complementada com referências dos artigos primários. Discussão: doenças da tireoide, como o hipotireoidismo e hipertireoidismo e doenças relacionadas às glândulas adrenais como Feocromocitoma, Doença de Addison e as Síndromes e Doença de Cushing podem ocasionar sintomas de ordem psiquiátrica, suprimindo sintomas já existentes ou intensificando os mesmos. Conclusão: pode-se perceber que o psiquiatra clínico deve manter atenção para as interfaces clínicas das doenças endócrinas uma vez que essas afecções podem mimetizar os quadros 
psiquiátricos mais comuns e saber como criar uma rotina de rastreio clínico e laboratorial para exclusão ou diagnóstico desses casos.

Palavras chave: Endocrinologia, Psiquiatria, Psiquiatria biológica

\begin{abstract}
:
Introduction: Several clinical syndromes can present neurological and psychiatric symptoms. Objective: This article is focused on describing the main endocrines syndromes witch can be developed with psychiatric disorders. Method: A literature review was carried out by searching and analyzing articles in English at Pubmed with descriptors and complemented with references from primary articles. Discussion: thyroid diseases as hypothyroidism and hyperthyroidism and diseases related to the adrenal glands as pheochromocytoma, Addison's disease and Cushing's syndromes and disease can cause psychiatric symptoms, either suppressing existing symptoms or intensifying them. Conclusion: it can be verified that the clinical psychiatrist must maintain attention to the clinical interfaces of endocrine diseases since these conditions can mimic the most common psychiatric conditions and must to know how to create a routine clinical and laboratory screening to exclude or diagnose these cases.
\end{abstract}

Keywords: Endocrinology, Psychiatry, Biological Psychiatry

\title{
RESUMEN:
}

Introducción: varios síndromes clínicos pueden presentar síntomas neurológicos y psiquiátricos. Objetivo: este artículo se centra en describir los principales síndromes endocrinos que pueden desarrollarse con los trastornos psiquiátricos. Método: Se realizó una revisión bibliográfica mediante la búsqueda y análisis de artículos en inglés en Pubmed con descriptores y complementada con referencias de artículos primarios. Discusión: las enfermedades tiroideas como el hipotiroidismo y el hipertiroidismo y las enfermedades relacionadas con las glándulas suprarrenales como el feocromocitoma, la enfermedad de Addison y los síndromes y enfermedades de Cushing pueden causar síntomas psiquiátricos, ya sea suprimiendo los síntomas existentes o intensificándolos. Conclusión: se puede verificar que el psiquiatra clínico 
debe mantener la atención a las interfases clínicas de las enfermedades endocrinas ya que estas condiciones pueden simular las condiciones psiquiátricas más comunes y debe saber crear un tamizaje clínico y de laboratorio de rutina para excluir o diagnosticar estos casos.

Palabras clave: Endocrinología, Psiquiatría, Psiquiatría Biológica

Como citar: Lafayette DAS, Urquiza PAC, Brito EMC, Costa SPGA, Cantilino A. - Interface de doenças endocrinológicas com repercussões psiquiátricas: uma revisão de literatura. Debates em Psiquiatria, Rio de Janeiro, 2021; 11:1-19. https://doi.org/10.25118/2763-

9037.2021.v11.271

Conflito de interesses: declaram não haver

Fonte de financiamento: declaram não haver

Parecer CEP: não se aplica

Recebido em: 30/11/2020

Aprovado em: 16/11/2021

Publicado em: 29/12/2021

\section{Introdução}

Várias síndromes clínicas podem apresentam sintomas neurológicos e psiquiátricos, e o diagnóstico precoce da causa primária pode ser decisivo para melhor gerenciamento de sintomas e superação da doença [1]. Este artigo está focado em descrever as principais síndrome endócrinas que podem cursar com alterações psiquiátricas.

\section{Método}

Foi realizada uma revisão de literatura, período de 2011 a 2021, por meio de busca e análise de artigos em inglês no pubmed com os seguintes descritores: (Mental Disorders OR Psychiatric Symptoms OR Psychiatric Complications OR Psychiatric Events OR neuropsychiatric events OR depression OR psychosis OR anxiety) AND (Cushing's syndrome OR Addison disease OR Adrenal insufficiency OR Hyperthyroidism OR Hypothyroidism OR Pheochromocytoma). A pesquisa foi complementada com referências dos artigos primários. 


\section{Doenças da tireoide}

A tireoide é uma glândula essencial para a regulação metabólica de todo o organismo através da conversão de seus hormônios T4 em T3 nos tecidos periféricos [2] . A triiodotironina (T3) alcança os receptores de T3 que estão espalhados por todo o cérebro, especialmente no sistema límbico dando suporte a várias funções, incluindo emoções e comportamento [ㅁ]. As principais alterações decorrentes das doenças tireoidianas são os estados de hiper ou hipotireoidismo que podem levar às alterações neuropsiquiátricas.

Um estudo com 2142 pessoas encontrou associação positiva de distúrbios da tireoide não tratados com depressão (RR 1.44 | 1.05 - 1.96) e ansiedade (RR $1.69 \mid 1.05$ - 2.72). O hipertireoidismo não tratado esteve relacionado com transtorno depressivo maior nos últimos doze meses (RR 3.58 | 1.34 - 9.57) enquanto o hipotireoidismo com maior risco de episódio depressivo atual pela Beck Depressive Inventory II que avalia sintomas depressivos nas últimas duas semanas (RR $2.32 \mid 1.28$ - 4.21). Nesse estudo, as avaliações do estado mental ocorreram de 1 a 5 anos após o diagnóstico do distúrbio tireoidiano o que pode sugerir que eles são precursores de depressão e ansiedade [4]].

Nesse contexto, sabe-se que comumente o hipotireoidismo mimetiza os sintomas de depressão, ansiedade e, mais raramente, sintomas maniatiformes; enquanto o hipertireoidismo mimetiza depressão, ansiedade, disforia, labilidade emocional e estados maniatiformes [ㄹ] .

\section{Hipotireoidismo}

O hipotireoidismo pode ocorrer em sua forma clínica (TSH aumentado e T4/T3 livres diminuídos) ou subclínica (apenas TSH aumentado). No hipotireoidismo ocorre sobreposição importante dos sintomas diagnósticos clínicos com os sintomas de depressão, à exceção de insônia que costuma ser mais frequente nos episódios depressivos [ㄹ] .

Uma metanálise mostrou que sintomas depressivos estiveram 3 vezes mais associados em pacientes com hipotiroidismo quando comparados com os controles (OR 3.31 95\% IC 2.00-5.48) numa amostra de 43.382 pacientes e cerca de 2 vezes mais associados com sintomas ansiosos (OR 2.32 95\% IC 1.40-3.85) numa amostra de 34.095 indivíduos, nesse estudo as tireoidites autoimunes eram a causa do hipotireoidismo. 
Os quadros ansiosos tendem a abrir com agitação, inquietação e taquicardia noturna. Além disso, também podem ocorrer prejuízos atencionais e queixa de fadiga []ㅡ. Nos pacientes com hipotireoidismo de longa data não tratados, apresentações psiquiátricas mais graves podem acontecer com depressão melancólica e mais raramente quadros psicóticos com delírios paranóides, alucinações audioverbais, afrouxamento das associações ou ideias deliroides, inclusive com relatos de casos de episódios maníacos associados ao hipotireoidismo [르, $\underline{6}]$.

O hipotireoidismo subclínico, definido como TSH elevado e T4 em níveis normais, tem uma prevalência de 3 a $8,5 \%$ da população geral. Apresentase com maior predominância no sexo feminino e nos idosos pode chegar a $20 \%$ do grupo []ㅡ. Essa entidade clínica é muito relacionada a sintomas depressivos além de outros sintomas psiquiátricos como nervosismo, irritabilidade e ansiedade com prejuízo na qualidade de vida [2] .

Duas metanálises recentes encontraram maior associação de depressão em pessoas com hipotireoidismo subclínico (OR 1.78, 95\% IC, 1.11-2.86, $p=0.02$ e RR 2.36, 95\% IC, $1.84-3.02 p<0.001$ ) sendo esse risco maior em pessoas acima de 50 anos $[\underline{7}, \underline{8}]$.

Um achado interessante foi que as pesquisas provenientes da Coreia do Sul, Noruega e Holanda não mostraram associação entre hipotireoidismo subclínico e depressão. Por outro lado, os três estudos brasileiros que entraram nessa metanálise mostraram positividade para esse desfecho, suscitando a ideia de que diferenças regionais podem influenciar [ㅁ].

Usualmente, os pacientes podem apresentar sintomas de intolerância ao frio, ganho de peso e disfunções cognitivas, sendo esses os sintomas que chamam a atenção para diagnóstico de hipotiroidismo, entretanto apenas $30 \%$ dos indivíduos com hipotireoidismo subclínico os apresentam []].

Normalmente, o tratamento do hipotireoidismo é feito com o uso de levotiroxina (T4) tendo como meta a normalização dos níveis de TSH. Com isso, é esperada melhora dos sintomas psiquiátricos, entretanto o padrão dessa melhora é incerto e a remissão dos sintomas ocorre de maneira variável entre os indivíduos. Mesmo após restabelecimento do estado eutireoideo, os indivíduos ainda podem persistir com sintomas neuropsiquiátricos [2]]. 
Quanto ao tratamento do hipotiroidismo subclínico, os guidelines não levam em consideração as manifestações psiquiátricas para indicar o uso de levotiroxina, sendo atualmente recomendado tratar quando $\mathrm{TSH}>10$, se há presença de anticorpos antitireoidianos, se há desejo de engravidar ou se há fatores de risco cardiovasculares associados (hipertensão e dislipidemia) $[\underline{2}, \underline{7}]$.

Aqueles pacientes que tratam depressão e tem hipotireoidismo subclínico associado ou apresentam TSH no limite superior da normalidade podem se beneficiar de associação do T3 ou T4. Apesar do TSH ser considerado normal até 4,5 mUI/ml, 90 a 95\% dos indivíduos tem TSH $<2,5$, sugerindo que TSH aferido repetidas vezes acima de $2,5 \mathrm{mUI} / \mathrm{ml}$ seja sinal de inadequação tireoidiana, sendo esse grupo associado a pior resposta aos antidepressivos [ㅁ] . Nesse sentido, pode ser indicada potencialização com T3 (25-50mcg/dia) ou T4 (50-100 mcg/dia) [9] ]. O uso de T4 deve ser priorizado se o paciente tem diagnóstico de hipotiroidismo subclínico. Os pacientes que responderam à suplementação diária com T3 tinham hormônios tireoidianos circulantes previamente mais baixos $\mathrm{e}$ experimentaram maior queda nos níveis do TSH [2] ]. Uma tentativa adequada requer pelo menos 6 semanas de uso, com alvo do TSH $<2,0$ e os efeitos totais podem levar até 6 meses para se consolidarem [ㅇ].

\section{Hipertireoidismo}

O hipertireoidismo é definido por TSH $<0,1 \mathrm{mUI} / \mathrm{ml}$ associado níveis elevados de T3 e T4, enquanto o hipertiroidismo subclínico apresenta apenas TSH reduzido. Nos estados tireotóxicos podem acontecer uma ampla variação nos sintomas neuropsiquiátricos que transitam de ansiedade (cerca de $60 \%$ dos pacientes hipertireoideos) à depressão. Em menor frequência se observa mania e mais raramente sintomas psicóticos com delírios persecutórios [2] ]. Sendo a maioria das descrições de psicose relacionadas à doença de Graves (hipertireoidismo com anticorpos TRab positivos) [ $[3]$. Muitos pacientes com hipertireoidismo acabam sendo encaminhados primeiro para avaliação de doença psiquiátrica primária e podem levar até 1 mês para um diagnóstico acurado [2] ]. No hipertireoidismo, usualmente, o paciente se apresenta com sintomas físicos de intolerância ao calor, sudorese excessiva, perda de peso, palpitações e desconforto gastrointestinal [ㅍ].

De forma mais intensa pode ocorrer uma tempestade tireoidiana, definida como a presença de sintomas graves e intensos daqueles já esperados 
para uma tireotoxicose. Sua frequência é rara, acontece em menos de $10 \%$ dos indivíduos que são internados com hipertireoidismo. Apresentam-se com taquicardia, fibrilação atrial, insuficiência cardíaca aguda, agitação, delirium, psicose, estupor, náusea, insuficiência hepática, hiperpirexia até coma. Envolvimento do SNC com delirium, psicose ou letargia nas tempestades tireoideanas estiveram associados com maior casuística fatal, o que chama a atenção para a gravidade desta entidade clínica que teve mortalidade de $25 \%$ numa série de 28 casos [10].

Nos casos de psicose associada a tireotoxicose, o que chama a atenção para o quadro é o início agudo dos sintomas psicóticos que estão cronologicamente relacionados com os sintomas físicos e a melhora dos sintomas psicóticos com o controle do hipertireoidismo (evidenciado pela melhora bioquímica e dos sinais vitais) []ㅡ.

O tratamento do hipertireoidismo deve ser realizado com medicações antitireoidianas adequadas [2]]. Em relação aos episódios psicóticos diagnosticados como secundários ao hipertireoidismo, uso de antipsicóticos atípicos ou típicos tiveram eficácia comparável entre si no manejo clínico, devendo a escolha ser baseada de acordo com o perfil do paciente e efeitos adversos das medicações [11].

Nos pacientes tratados para hipertireoidismo, apenas $50 \%$ tiveram recuperação completa dos sintomas neuropsiquiátricos, cerca de $35 \%$ tiveram recuperação parcial e $15 \%$ não obtiveram mudança no seu estado mental após recuperação do estado bioquímico; eles persistiram com mais sintomas depressivos, ansiosos, alteração no sono, déficits de memória, de atenção e executivo. Quanto mais tempo em hipertiroidismo, maior a probabilidade de sintomas neuropsiquiátricos residuais [2]].

Devido à inespecificidade dos sintomas neuropsiquiátricos e grande associação dos distúrbios psiquiátricos com os distúrbios tireoidianos, é interessante o rastreio da função tiroidiana (TSH e T4 Livre) e dos anticorpos antitireoidianos (Anti-TPO e TRab) para todos os pacientes que tenham sintomas psiquiátricos $[\underline{2}, \underline{5}]$.

\section{Doenças relacionadas à glândula adrenal Feocromocitoma}

Feocromocitoma é uma doença tumoral incomum das células cromafins da medula adrenal que acomete igualmente homens e mulheres, entre 20 e 50 anos. Sua incidência anual é de 2 a 8 casos/milhão. Tem como sua característica básica a produção de catecolaminas (adrenalina, 
noradrenalina ou dopamina) e devido a isso deriva sua apresentação clínica, tendo como sinal mais comum a hipertensão. É considerado a partir de uma tríade clássica de cefaléia pulsátil, sudorese e taquicardia, apesar de ser difícil encontrar esses três sintomas na maioria dos diagnósticos. Apresenta-se em liberações episódicas causando sintomas paroxísticos [12].

Devido a essa dificuldade diagnóstica e a relativa raridade de sua apresentação é considerado pelos descritores como "O grande mímico" pois apresenta muitos diagnósticos diferenciais e sintomatologia variada. Entre esses sintomas estão as manifestações psiquiátricas. O primeiro caso descrito de sintomatologia psiquiátrica no feocromocitoma apresentava uma mulher que abriu o quadro com um estado de ansiedade aguda com algumas semanas de duração que apresentava associado a isso manifestações somáticas com agitação, tremores, palpitações, náusea e sudorese em ataques que duravam cerca de 10 minutos o que chama a atenção para a similaridade com ataques de pânico, viu-se que essa apresentação paroxística se repetia em vários indivíduos [13]. Já outros pacientes apresentam sintomas mais contínuos que chamavam a atenção para diagnóstico diferencial com Transtorno de Ansiedade Generalizada. Esses pacientes apresentam mais sintomas relacionados à esfera ansiosa e em menor grau à depressiva.

Menos frequentemente, os pacientes podem evoluir para síndromes psicóticas francas associadas a quadros ansiosos e sintomas físicos, a título de relato de casos foram utilizados antipsicóticos atípicos com resposta controversa. Em um paciente havia resposta aos antipsicóticos, porém a psicose recorreu 8 vezes em 2 anos com melhora após redução das catecolaminas. Em outro paciente, a melhora também só veio concomitante a redução plasmática das catecolaminas. O psiquiatra deve estar atento a presença de sintomas mentais associados a instabilidade autonômica com hipertensão para suspeitar do diagnóstico [14].

Tendo isso em alerta, deve-se solicitar a coleta urinária $(>1,8 \mathrm{mg} / 24 \mathrm{~h})$ e plasmática ( $>=2000 \mathrm{pg} / \mathrm{ml}$ ) de metanefrinas que em combinação traz uma acurácia de cerca de $98 \%$. Mas com dosagem plasmática já acima de $1000 \mathrm{pg} / \mathrm{ml}$ a suspeita deve ser alta. Após o diagnóstico laboratorial é necessária a localização do tumor que é feita por ressonância magnética de abdômen. 
Deve-se ter em mente que tricíclicos, ciclobenzaprina, anfetaminas e a retirada de clonidina podem elevar as metanefrinas no curto prazo assim como cafeína e cigarro (o paciente não pode usar essas substâncias a pelo menos $24 \mathrm{~h}$ da coleta) [15].

\section{Doença de Addison}

A doença de Addison é o resultado da destruição do córtex da suprarrenal, levando, principalmente, à redução de glico e mineralocorticóides [16].

A etiologia mais comum é a adrenalite autoimune, mas causas infecciosas (tuberculose, fungos, HIV e sífilis), infartos da glândula após choques hipovolêmicos e outras doenças autoimunes podem dar origem ao quadro $[17,18]$. Os sintomas clínicos são variados e inespecíficos: perda de peso, mialgia e artralgias, perda de pelos, amenorreia, náusea e vômitos sem causa específica, dor abdominal e alterações do hábito intestinal. No entanto, a suspeita principal deve ser de hipoglicemia e hiponatremia sustentada (devido à perda de mineralocorticoides), associada à hiperpigmentação da pele e superfícies mucosas, pois o Hormônio Estimulador do Córtex Adrenal (ACTH) é estimulado e é co-secretado com o Hormônio Melanotrófico. Pacientes com vitiligo devem ser melhor acompanhados, devido maior risco de doença adrenal autoimune [17, 19]. No que diz respeito aos sintomas psiquiátricos, são mais encontrados: fadiga, perda da libido, sintomas depressivos (mais comum), irritabilidade, distúrbios do sono, apatia, comprometimento cognitivo e alucinações [ $\underline{20}$, 21]. A síndrome psicótica (incluindo delírio e catatonia) é rara e é mais encontrada na doença com apresentação grave $[\underline{22}, \underline{23}]$.

São encontrados, no cérebro, receptores de glicorticóides, sendo mais comuns no hipocampo [24]. Em estudo recente, este hormônio também foi importante para a manutenção do funcionamento do córtex pré-frontal [25]. A redução abrupta de corticóides pode resultar em morte celular hipocampal e no giro denteado, levando ao comprometimento da memória e da cognição [르].

O diagnóstico da doença de Addison é dado após análise clínica, sob suspeita, dosa-se o cortisol plasmático no horário das 10 horas, onde pode vir com níveis plasmáticos baixos e com o ACTH elevado, caso seja este o resultado, será realizado novo teste de estimulação com corticotropina a 250mg (análogo do ACTH), aplicada às 10 horas da manhã e dosado, 60 minutos depois, os níveis de cortisol. Em pacientes com doença de Addison, - córtex adrenal é incapaz de aumentar a secreção de hormônio à estimulação [26, 27]]. 
O tratamento deve abordar não apenas a reposição de glicocorticóides, mas também as outras condições clínicas presentes (hiponatremia, hipoglicemia). A reposição hormonal, em quadro agudo, pode ser feita, preferencialmente, com dexametasona $4 \mathrm{mg}$ EV em bolus. Cronicamente, a reposição com dexametasona ou prednisona poderá ser abordada. Para casos com distúrbios hidroeletróliticos a fludrocortisona deve ser avaliada, por ter maior efeito similar ao mineralocorticoide [28-31]. A reposição deve ser controlada, buscando-se a menor dose possível para o bem-estar do paciente [29].

\section{Síndrome e Doença de Cushing}

A Doença de Cushing é caracterizada por um aumento da produção do Hormônio Adrenocorticotrófico (ACTH), geralmente por um tumor localizado na hipófise, levando à superprodução de glicocorticóides pelo córtex da Adrenal [32]. A Síndrome de Cushing (SC) é vista pelo aumento generalizado deste hormônio, mas resultante também por outras vias (como uso externo via medicação). Clinicamente, a síndrome pode vir a se apresentar por: hipertensão, fraqueza muscular, distribuição anormal de gordura corporal, osteoporose, hirsutismo e disfunção gonadal. Juntamente, sintomas psiquiátricos podem vir associados: depressão (pode ocorrer em $50-60 \%$ dos pacientes, potencialmente fatal), déficits cognitivos (parciais e/ou definitivos), apatia, ansiedade, mania, e psicose $[\underline{32}, \underline{33}]$.

Em estudos com Ressonância Magnética, na fase ativa da SC, foram encontrados menores volumes cerebrais, ventrículos maiores, volume menor de amígdala, menor volume hipocampal, redução do volume do cerebelo, associado a processos desmielinizantes. Após um ano de tratamento, ocorreu uma tendência ao aumento do volume cerebral, à redução do tamanho ventricular e ao aumento do volume do hipocampo [34-38]. Após a remissão da doença, os sintomas podem melhorar consideravelmente $[\underline{2}, \underline{39}, \underline{40}]$. No entanto, mesmo após remissão da doença a dificuldade cognitiva persistiu, assim como a depressão e a ansiedade, resultando em redução da qualidade de vida [노, $\underline{41}, \underline{42}]$.

A maioria dos pacientes com doença de Cushing é tratada por meio de cirurgia transesfenoidal, em alguns casos seguido de radioterapia pósoperatória, tratamento farmacológico e/ou adrenalectomia bilateral, dependendo da eficácia da cirurgia $[\underline{30}, \underline{43}]$. 
Quanto aos sintomas psiquiátricos, seria prioritário aguardar a normalização dos níveis de cortisol, antes de prescrever um psicofármaco, pois os pacientes podem não responder adequadamente a algumas classes de medicamentos (Inibidores Seletivos da Recaptação da Serotonina parecem ter efeito parcial, enquanto Antidepressivos Tricíclicos têm pouco ou nenhum efeito) [44, 45]. Para a SC com sintomas psicóticos graves é possível considerar antipsicóticos atípicos como sintomáticos [46].

Considerando, o acompanhamento a longo prazo, uma investigação contínua de sintomas psiquiátricos deve ser continuada, mesmo com a doença superada [33]. O acompanhamento psicoterápico pode ser útil, principalmente na presença de transtornos afetivos $[\underline{47}, \underline{48}]$.

\section{Conclusão}

Nesse sentido, pode-se perceber que o psiquiatra clínico deve manter atenção para as interfaces clínicas das doenças endócrinas uma vez que essas afecções podem mimetizar os quadros psiquiátricos mais comuns. Sendo assim, o psiquiatra deve criar uma rotina de rastreio clínico e laboratorial para tentar excluir esses casos.

\section{Agradecimentos}

A todos nossos preceptores, enfermeiros, técnicos e equipe do Serviço de Psiquiatria, sem os quais nada seríamos. 


\title{
Referências
}

- 1. Isaac ML, Larson EB. Medical conditions with neuropsychiatric Manifestations. Med Clin North Am. 2014 Sep; 98(5):1193-208. http://dx.doi.org/10.1016/j.mcna.2014.06.012 - PMid:25134879

\begin{abstract}
2. Feldman AZ, Shrestha RT, Hennessey JV. Neuropsychiatric Manifestations of Thyroid Disease. Endocrinology and Metabolism Clinics of North America. 2013.

https://doi.org/10.1016/j.ecl.2013.05.005 - PMid:24011880
\end{abstract}

3. Lee KA, Park KT, Yu HM, Jin HY, Baek HS, Park TS. Subacute thyroiditis presenting as acute psychosis: a case report and literature review. Korean J Intern Med [Internet]. 2013 Mar [cited 2019 Jul 23];28(2):242-6. Available from:

http://www.ncbi.nlm.nih.gov/pubmed/23526704 https://doi.org/10.3904/kjim.2013.28.2.242 - PMid:23526704 PMCid:PMC3604616

4. Ittermann T, Völzke H, Baumeister SE, Appel K, Grabe HJ. Diagnosed thyroid disorders are associated with depression and anxiety. Soc Psychiatry Psychiatr Epidemiol [Internet]. 2015 Sep [cited 2019 Jul 23];50(9):1417-25. Available from:

http://www.ncbi.nlm.nih.gov/pubmed/25777685 https://doi.org/10.1007/s00127-015-1043-0 - PMid:25777685

5. Siegmann EM, Müller HHO, Luecke C, Philipsen A, Kornhuber J, Grömer TW. Association of depression and anxiety disorders with autoimmune thyroiditis: A systematic review and meta-analysis. JAMA Psychiatry.2018.

https://doi.org/10.1001/jamapsychiatry.2018.0190 -

PMid:29800939 PMCid:PMC6137529

6. Khemka D, Ali JA, Koch CA. Primary hypothyroidism associated with acute mania: Case series and literature review. Experimental and Clinical Endocrinology and Diabetes. 2011.

https://doi.org/10.1055/s-0031-1277137 - PMid:21553358

12 Debates em Psiquiatria, Rio de Janeiro, 2021; 11:1-19 https://doi.org/10.25118/2763-9037.2021.v11.271 
7. Loh HH, Lim LL, Yee A, Loh HS. Association between subclinical hypothyroidism and depression: An updated systematic review and meta-analysis. BMC Psychiatry. 2019.

https://doi.org/10.1186/s12888-018-2006-2 - PMid:30621645 PMCid:PMC6325749

8. Tang R, Wang J, Yang L, Ding X, Zhong Y, Pan J, et al. Subclinical Hypothyroidism and Depression: A Systematic Review and MetaAnalysis. Front Endocrinol (Lausanne). 2019.

https://doi.org/10.3389/fendo.2019.00340 - PMid:31214119 PMCid:PMC6558168

9. Cohen BM, Sommer BR, Vuckovic A. Antidepressant-resistant diarréia diarréia depression in patients with comorbid subclinical hypothyroidism or high-normal TSH levels. Am J Psychiatry. 2018. https://doi.org/10.1176/appi.ajp.2017.17080949 - PMid:29961367

10. Swee DS, Chng CL, Lim A. Clinical characteristics and outcome of thyroid storm: a case series and review of neuropsychiatric derangements in thyrotoxicosis. Endocr Pract [Internet]. 2015 Feb [cited 2019 Jul 23];21(2):182-9. Available from:

http://www.ncbi.nlm.nih.gov/pubmed/25370315 https://doi.org/10.4158/EP14023.OR - PMid:25370315

11. Golub D, Rodack V. Antipsychotics in Hyperthyroid-Related

Psychosis: Case Report and Systematic Review. Neuro Endocrinol Lett [Internet]. 2018 Mar [cited 2019 Aug 23];39(1):65-74.

Available from: http://www.ncbi.nlm.nih.gov/pubmed/29803209

12. Gunawardane PTK, Grossman A. Phaeochromocytoma and Paraganglioma. Adv Exp Med Biol [Internet]. 2017 [cited 2019 Jul 24];956:239-59. Available from:

http://www.ncbi.nlm.nih.gov/pubmed/27888488 https://doi.org/10.1007/55842016 76 - PMid:27888488

13. Medvei VC, Cattell WR. Mental symptoms presenting in phaeochromocytoma: a case report and review. J R Soc Med [Internet]. 1988 Sep [cited 2019 Jul 24];81(9):550-1. Available from: http://www.ncbi.nlm.nih.gov/pubmed/3054110 
https://doi.org/10.1177/014107688808100923 - PMid:3054110 PMCid:PMC1291775

14. Brown JS. Cases of remission of psychosis following resection of pheochromocytoma or paraganglioma. Schizophr Res. 2016.

https://doi.org/10.1016/j.schres.2016.08.012 - PMid:27534680

15. Leung A, Zun L, Nordstrom K, Wilson MP. Psychiatric

Emergencies for Physicians: Clinical Management and Approach to Distinguishing Pheochromocytoma From Psychiatric and Thyrotoxic Diseases in the Emergency Department. J Emerg Med [Internet]. 2017 Nov [cited 2019 Jul 24];53(5):712-6. Available from: http://www.ncbi.nlm.nih.gov/pubmed/28874302 https://doi.org/10.1016/j.jemermed.2017.05.032 - PMid:28874302

16. Addison T: On the Constitutional and Local Effects of Disease of the Suprarenal Capsules. Birmingham, Ala, Classics of Medicine Library, 1980

17. Oelkers W: Adrenal Insufficiency. N Engl J Med 1996; 335:12061212. https://doi.org/10.1056/NEJM199610173351607 PMid: 8815944

18. Eisenbarth GS, Gottlieb PA. Autoimmune polyendocrine syndromes. N Engl J Med 2004; 350:2068-2079. https://doi.org/10.1056/NEJMra030158 - PMid:15141045

19. Dekkers OM, van der Klaauw AA, Pereira AM, Biermasz NR, Honkoop PJ, Roelfsema F, Smit JW, Romijn JA. Quality of life is decreased after treatment for nonfunctioning pituitary macroadenoma. J Clin Endocrinol Metab 2006; 91:3364-3369. https://doi.org/10.1210/jc.2006-0003 - PMid:16787991

20. Løvas K, Husebye ES. High prevalence and increasing incidence of Addison's disease in western Norway. Clin Endocrinol (Oxf). 2002; 56:787-91. https://doi.org/10.1046/j.1365-2265.2002.t01-101552.X - PMid:12072049

21. Erichsen $M M, L ø v a{ }^{\circ} s K$, Skinningsrud $B$, Wolff $A B$, Undlien $D E$, Svartberg J, Fougner KJ, Berg TJ, Bollerslev J, Mella B, Carlson JA, Erlich $\mathrm{H}$, Husebye ES. Clinical, immunological, and genetic features 
of autoimmune primary adrenal insufficiency: observations from a Norwegian registry. J Clin Endocrinol Metab. 2009;94:4882-90. https://doi.org/10.1210/jc.2009-1368 - PMid:19858318

22. Thomsen AF, Kvist TK, Andersen PK, Kessing LV. The risk of affective disorders in patients with adrenocortical insufficiency. Psychoneuroendocrinology. 2006;31:614-22. https://doi.org/10.1016/j.psyneuen.2006.01.003 - PMid:16545526

23. Holtzman CW, Trotman HD, Goulding SM, Ryan AT, MacDonald AN, Shapiro DI, Brasfield JL, Walker EF. Stress and neurodevelopmental processes in the emergence of psychosis. Neuroscience. 2013;249:172-91. https://doi.org/10.1016/j.neuroscience.2012.12.017 PMid:23298853 - PMCid:PMC4140178

24. Gould E, Woolley CS, McEwen BS. Short-term glucocorticoid manipulations affect neuronal morphology and survival in the adult dentate gyrus. Neuroscience 1990; 37:367-375. https://doi.org/10.1016/0306-4522(90)90407-U

25. Squire LR, Zola-Morgan S. Memory: brain systems and behavior. Trends Neurosci 1988. https://doi.org/10.1016/01662236(88)90144-0

26. Mizoguchi K, Ishige A, Takeda S, Aburada M, Tabira T. Endogenous glucocorticoids are essential for maintaining prefrontal cortical cognitive function. J Neurosci 2004; 24:5492-5499. https://doi.org/10.1523/JNEUROSCI.0086-04.2004 PMid:15201321 - PMCid:PMC6729338

27. Filipsson $H$, Monson JP, Koltowska-Haggstrom $M$, Mattsson $A$, Johannsson $\mathrm{G}$. The impact of glucocorticoid replacement regimens on metabolic outcome and comorbidity in hypopituitary patients. J Clin Endocrinol Metab 2006;91:3954-3961. https://doi.org/10.1210/jc.2006-0524 - PMid:16895963

28. Johannsson $G$, Falorni A, Skrtic S, Lennernas $H$, Quinkler $M$, Monson JP, Stewart PM. Adrenal insufficiency: review of clinical outcomes with current glucocorticoid replacement therapy. Clin 
Endocrinol (Oxf) 2015; 82:2-11. https://doi.org/10.1111/cen.12603 PMid:25187037

29. Bornstein SR, Allolio B, Arlt W, et al. Diagnosis and Treatment of Primary Adrenal Insufficiency: An Endocrine Society Clinical Practice Guideline. J Clin Endocrinol Metab 2016; 101:364.

https://doi.org/10.1210/jc.2015-1710 - PMid:26760044 PMCid:PMC4880116

30. Grossman AB. Clinical Review: The diagnosis and management of central hypoadrenalism. J Clin Endocrinol Metab 2010; 95:4855. https://doi.org/10.1210/jc.2010-0982 - PMid:20719838

31. Anglin RE, Rosebush PI, Mazurek MF. The Neuropsychiatric Profile of Addison's Disease: Revisiting a Forgotten Phenomenon. J Neuropsychiatry Clin Neurosci. 2006;18(4):450-9. http://doi.org/10.1176/jnp.2006.18.4.450 - PMID:17135373

32. Newell-Price J, Bertagna X, Grossman AB, Nieman LK. Cushing's syndrome. Lancet 2006;367:1605-1617. https://doi.org/10.1016/S0140-6736(06)68699-6

33. Pivonello R, Simeoli C, De Martino MC, Cozzolino A, De Leo M, Iacuaniello D, Pivonello C, Negri M, Pellecchia MT, Iasevoli F, Colao A. Neuropsychiatric disorders in Cushing's syndrome. Front. Neurosci. 2015,9,129. https://doi.org/10.3389/fnins.2015.00129 PMid:25941467 - PMCid:PMC4403344

34. Merke DP, Giedd JN, Keil MF, Mehlinger SL, Wiggs EA, Holzer S, Rawson E, Vaituzis AC, Stratakis CA, Chrousos GP. Children experience cognitive decline despite reversal of brain atrophy one year after resolution of Cushing syndrome. J Clin Endocrinol Metab 2005;90:2531-2536. https://doi.org/10.1210/jc.2004-2488 PMid: 15741254

35. Bauduin SEEC, van der Wee NJA, van der Werff SJA. Curr Opin Endocrinol Diabetes Obes. 2018 Aug;25(4):285-289. https://doi.org/10.1097/MED.0000000000000414 - PMid:29746308

36. Simmons NE, Do HM, Lipper MH, Laws ER Jr. Cerebral atrophy in Cushing's disease. Surg Neurol 2000; 53:72-76 
https://doi.org/10.1016/S0090-3019(99)00197-4

37. Resmini E, Santos A, Gomez-Anson B, Vives Y, Pires P, Crespo I, Portella MJ, Juan-Delago M, Barahona MJ, Webb SM. Verbal and visual memory performance and hippocampal volumes, measured by 3-Tesla magnetic resonance imaging, in patients with Cushing's syndrome. J Clin Endocrinol Metab 2012; 97:663-671. https://doi.org/10.1210/jc.2011-2231 - PMid:22162471

38. Santos A, Resmini E, Crespo I, Pires P, Vives-Gilabert Y, Granell E, Valassi E, Gómez-Anson B, Martínez-Momblán MA, Mataró M, Webb SM. Small cerebellar cortex volume in patients with active Cushing's syndrome. Eur J Endocrinol 2014; 171:461-469. https://doi.org/10.1530/EJE-14-0371 - PMid:25005936

39. Cohen SI. Cushing's syndrome: a psychiatric study of 29 patients. Br J Psychiatry 1980; 136:120-124.

https://doi.org/10.1192/bjp.136.2.120 - PMid:7370477

40. Kelly WF, Kelly MJ, Faragher B. A prospective study of psychiatric and psychological aspects of Cushing's syndrome. Clin Endocrinol (Oxf) 1996; 45:715-720.

https://doi.org/10.1046/j.1365-2265.1996.8690878.x PMid: 9039337

41. Tiemensma J, Biermasz NR, Middelkoop HA, van der Mast RC, Romijn JA, Pereira AM. Increased prevalence of psychopathology and maladaptive personality traits after long-term cure of Cushing's disease. J Clin Endocrinol Metab 2010;95:E129-E141. https://doi.org/10.1210/jc.2010-0512 - PMid:20660031

42. Tiemensma J, Kokshoorn NE, Biermasz NR, Keijser BJSA, Wassenaar MJE, Middelkoop HAM, Pereira AM, Romijnet JA. Subtle cognitive impairments in patients with long-term cure of Cushing's disease. J Clin Endocrinol Metab 2010;95:2699-2714. https://doi.org/10.1210/jc.2009-2032 - PMid:20371667

43. Nieman LK, Biller BM, Findling JW, Murad MH, Newell-Price J, Savage MO, Tabarinet A. Treatment of Cushing's syndrome: an Endocrine Society clinical practice guideline. J Clin Endocrinol Metab. 
2015;100:2807-31. https://doi.org/10.1210/jc.2015-1818 PMid:26222757 PMCid:PMC4525003

44. Sonino N, Fallo F, Fava GA. Psychosomatic aspects of Cushing's syndrome. Rev Endocr Metab Disord. 2010;11:95-104. https://doi.org/10.1007/s11154-009-9123-7 - PMid:19960264

- 45. Starkman MN. Neuropsychiatric findings in Cushing's syndrome and exogenous glucocorticoid administration. Endocrinol Metab Clin N Am. 2013;42:477-88. https://doi.org/10.1016/j.ecl.2013.05.010 PMid:24011881

- 46. Santos A, Resmini E, Pascual JC, Crespo I, Webb SM. Psychiatric Symptoms in Patients with Cushing's Syndrome: Prevalence, Diagnosis and Management. Drugs. 2017 May;77(8):829-842. https://doi.org/10.1007/s40265-017-0735-z - PMID:28393326.

47. Sonino N, Fava GA. Psychiatric disorders associated with Cushing's syndrome: epidemiology, pathophysiology and treatment. CNS Drugs. 2001;15:361-73. https://doi.org/10.2165/00023210200115050-00003 - PMid: 11475942

48. Dorn LD, Burgess ES, Friedman TC, Dubbert B, Gold PW, Chrousos GP. The longitudinal course of psychopathology in Cushing's syndrome after correction of hypercortisolism. J Clin Endocrinol Metab. 1997;82:912-9. https://doi.org/10.1210/jc.82.3.912 https://doi.org/10.1210/jcem.82.3.3834 - PMid: 9062506 
Daniell de Siqueira Araújo Lafayette

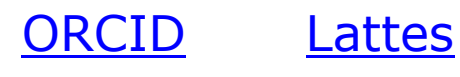

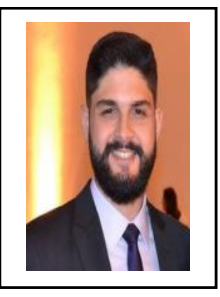

Philip de Azevedo Costa Urquiza

$\underline{\text { ORCID Lattes }}$

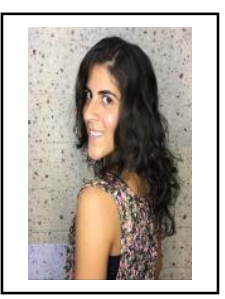

Eveline Maria Cosmo de Brito

$\underline{\text { ORCID } \quad \text { Lattes }}$

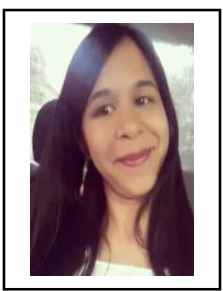

Silvia Poliana Guedes Alcoforado Costa

$\underline{\text { ORCID Lattes }}$

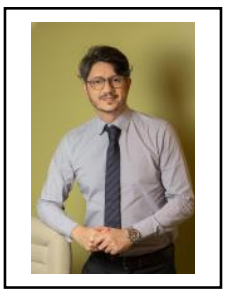

Amaury Cantilino

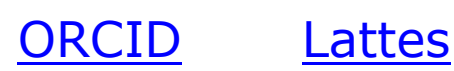

